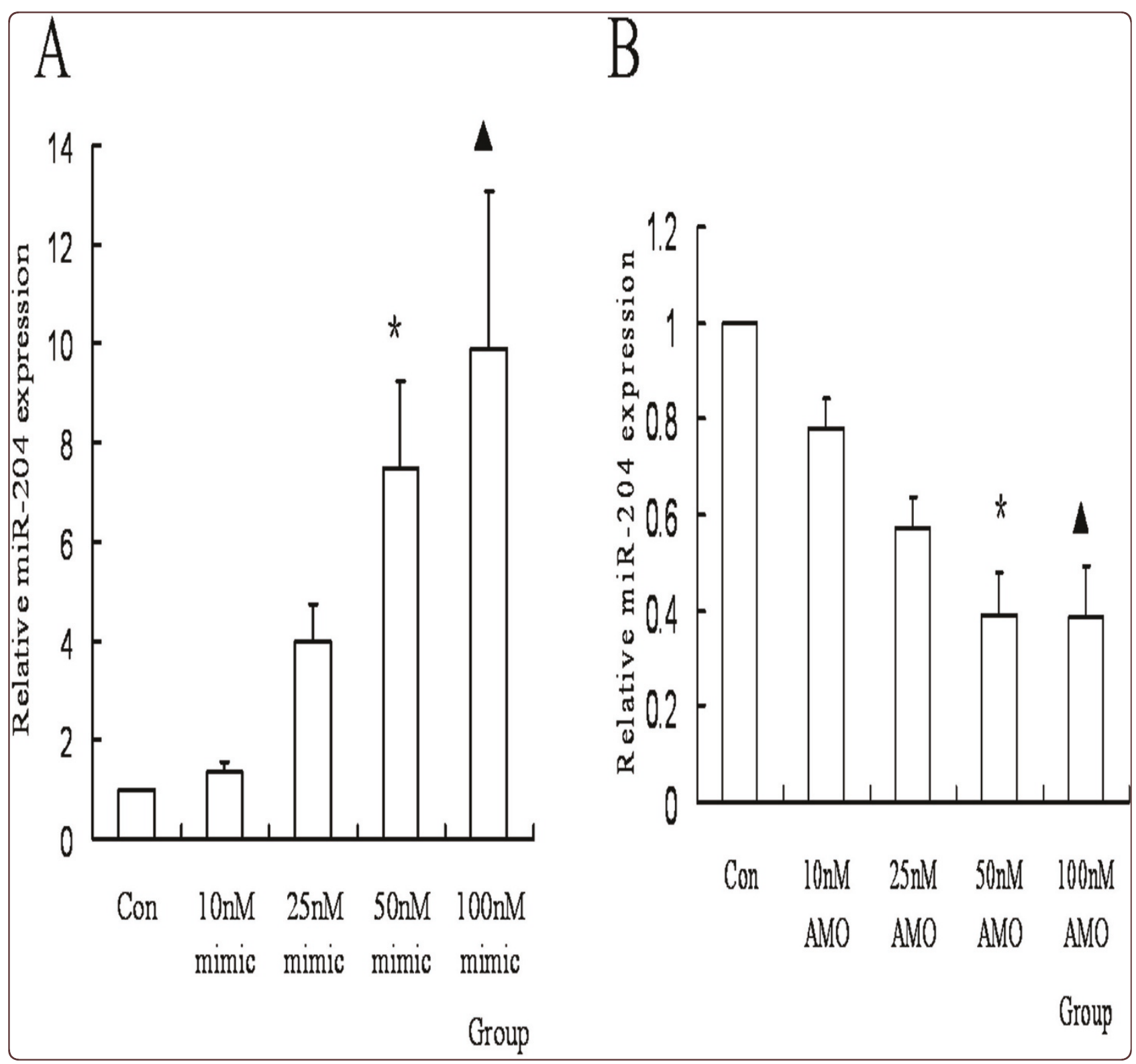

MiR-204 regulates cardiomyocyte autophagy
induced by ischemia-reperfusion through LC3-II

Xiao et al. 


\title{
MiR-204 regulates cardiomyocyte autophagy induced by ischemia-reperfusion through LC3-II
}

\author{
Jian Xiao ${ }^{1+}$, Xiaoyan Zhu ${ }^{2+}$, Bin $\mathrm{He}^{3 \dagger}$, Yufeng Zhang ${ }^{1}$, Bo Kang ${ }^{1}$, Zhinong Wang ${ }^{1^{*}}$ and Xin $\mathrm{Ni}^{2^{*}}$
}

\begin{abstract}
Background: Autophagy plays a significant role in myocardial ischemia-reperfusion (IR) injury. So it is important to inhibit autophagy to protect cardiomyocytes besides anti-apoptosis. MiRNA has been demonstrated to protect cardiomyocytes against apoptosis during $\mathrm{IR}$, while whether it has anti-autophagy effect has not been known. The aim of this study was to investigate whether miR-204 regulated autophagy by regulating LC3-II protein, which is the marker of autophagosome during myocardial IR injury.
\end{abstract}

Methods: Adult SD rats were randomized to Control and IR groups. IR group was treated with 30 min ischemia by ligating the left anterior descending coronary artery, followed by $2 \mathrm{~h}$ reperfusion by loosing the ligation. The expression of miR-204 was measured by RT-PCR, and LC3 protein was measured by western-blot.

Results: We found that IR induced cardiomyocytes autophagy, together with down-regulation of miR-204 and upregulation of LC3-II protein. And, we have found that LC3-II protein was regulated by miR-204, using the method of transferring miR-204 mimic or AMO-204 into the cardiomyocytes, before.

Conclusions: These studies provided evidence that miR-204 played an important role in regulating autophagy through LC3-IIprotein during IR.

\section{Background}

Autophagy is a type of programmed cell death. It has been suggested to be essential for cell homeostasis [1-3]. It can determine the cell survival together with apoptosis and necrosis $[4,5]$. Autophagy level is very low in physiological conditions, and is upregulated in many pathophysiological processes [6,7]. Because cardiomyocytes are terminally differentiated cells which can not divide again, suitable autophagy is essential for the maintenance of cardiomyocytes homeostasis. So, autophagocytic deficiencies or excess is associated with many cardiac pathologies, such as ischemia, IR, and heart failure [8,9]. It has been found that autophagy increased after IR [10], but it is still unclear whether autophagy protects the heart against IR injury or contributes to cell death. It seems that modest levels of autophagy appear to be protective. While high levels of

\footnotetext{
* Correspondence: wangzn007@hotmail.com; nixinphysiology@hotmail.com + Contributed equally

'Department of Cardiothoracic Surgery, Changzheng Hospital, the second military medical university, Fengyang road 415\#, shanghai, China, 200003 ${ }^{2}$ Department of Physiology, the second military medical university, Xiangyin road 800\#, Shanghai, China, 200433

Full list of author information is available at the end of the article
}

autophagy may cause self-digestion and promote cell death [11].

Autophagy is regulated by many autophagy related genes (Atgs) which are involved in autophagosome formation [12]. Among these Atgs, LC3 (microtubule-associated protein 1 light chain 3, Atg8) is localized on the autophagosome membrane. So LC3 is essential for the formation of autophagosome [13]. During the formation of autophagosome, the soluble form of LC3 (LC3-I) is convered to the autophagic vesicle-associated form (LC3-II), which is an important marker of autophagy [14]. So it is possible to control the process of autophagy by up-regulating or down-regulating LC3, and the molecular mechanism for this effect has yet to be elucidated.

As we know, microRNAs (miRNAs or miRs), which negatively regulate protein expression in diverse biological and pathological processes, have been demonstrated to play an important role in myocardial injury [15-17]. It has been observed that many miRNAs regulate cell apoptosis, such as miR-1, miR-133, miR-199, miR-208, miR-320, miR-21, and miR-204, etc [18-23]. However, it is well known that when apoptosis is blocked, the cells, which preferentially die by apoptosis, may die by autophagy [24].

\section{Biomed Central}


So it will be beneficial for cell survival if autophagy is inhibited together with apoptosis. We found that miR-204, which has anti-apoptosis effect, may also regulate LC3 expresion through the 9 complementary bases, according the bioinformatics of Targetscan.

So the present study was undertaken to see whether miR-204 was dysregulated by ischemia-reperfusion (IR), and if it may inhibit autophagy during hypoxiareoxygenation by regulating $\mathrm{LC}$.

\section{Material and methods}

\section{Animal care}

All animal experiments were approved by the Animal Research Ethics Committee of the Second Military Medical University, Shanghai, China. The investigation conformed with the guide for the care and use of laboratory animals published by the US National Institutes of Health.

\section{IR model and experimental protocols}

SD rats (250-300 g) were anesthetized with $10 \%$ chloral hydrate $(300 \mathrm{mg} / \mathrm{kg}$, i.p.) before endotracheal intubation. IR was induced by ligating the left anterior descending artery (LAD) for $30 \mathrm{~min}$, followed by loosening the ligature for $120 \mathrm{~min}$. Successful ligation of LAD was evidenced by immediate regional cyanosis in the anterior ventricular wall and the apex of the heart with color change greater than $40 \%$ of the left ventricle (LV) and confirmed by electrocardiography(ECG).

\section{Experimental protocols}

Twenty rats were equally randomly assigned into two groups: Control group (Con group, $n=10$ ), where the rats underwent thoracotomy without ligation; IR group $(n=10)$, where the rats were treated with ischemia for $30 \mathrm{~min}$ and reperfusion for $120 \mathrm{~min}$.

\section{Infarct size measurement}

Infarct size of the myocardium was measured as previously described. Infarct area (INF) and area at risk (AAR) were determined by computerized planimetry. The percentage of INF/AAR was calculated.

\section{LDH assay}

Blood serum was collected after 180 min reperfusion for determination of lactate dehydrogenase (LDH).

\section{Quantitative real-time RT-PCR of miR-204}

Total RNA of cells was isolated by using TRIzol reagent, and reverse transcribed according to the manufacturer's instructions (Fermentas, in CA). The annealing temperature of miRNA-204 was set at $60^{\circ} \mathrm{C}$. The comparative $\mathrm{Ct}$ (threshold cycle) method with arithmetic formulae $\left(2^{-\Delta \Delta \mathrm{Ct}}\right)$ was used to determine relative quantitation of gene expression of both target and housekeeping genes $(\beta$-actin). The primers of miR-204 used in the study are shown in Table 1.

\section{Western-blotting}

Protein concentration was determined with BCA protein assay kit according the manufacturer's protocol. Equal amounts of protein (60 ug) from the cardiomyocytes were subjected to Western-blotting analysis to evaluate LC3 expression with ECL detection kit (Amersham Biosciences, Piscataway, NJ). LC3 immunoreactivity was detected using a rabbit antiserum specific for rat Beclin1 protein (Sigma, USA) as primary antibodies. Detection of antigen-antibody complex formation was performed with horseradish peroxidase (HRP)-conjugated goat anti-rabbit secondary antibody. The LC3 concentrations were normalized with $\beta$-actin.

\section{Statistical analysis}

Quantitative data are presented as mean \pm standard error. Statistical significance was determined using $\mathrm{T}$ test or one-way ANOVA. $\mathrm{P}<0.05$ was considered statistically significant.

\section{Results}

Myocardium injury was induced by IR

The extent of myocardial infarction was evaluated after reperfusion. Representative photographs of midventricular cross sections of evans blue and TTC-stained hearts were taken from Control and IR groups. INF/AAR and LDH were shown in Figure 1.

\section{IR decreased the expression of miR-204}

To demonstrate the effect of IR on miR-204, we compared the miR-204 between the control group and IR group $(n=10)$. It was found that IR significantly decreased miR-204 with the method of Real-time PCR. (Figure 2)

\section{IR up-regulated the protein level of LC3-II}

As a marker of autophagosome, the protein level of LC3-II represents the amount of autophagosome. So we compared the ratio of LC3-II/LC3-I between the control

Table 1 Primers used for quantitative real-time RT-PCR

\begin{tabular}{ccc}
\hline Type & Name & Sequence \\
\hline RT-primer & miR-204 & 5'-GTCGTATCCAGTGCAGGGTCCGAGGTA \\
& & TICGCACTGGATACGACCCTTCTAGGCAT-3' \\
PCR-primer & miR204-F & 5'-CTGTCACTCGAGCTGCTGGAATG-3' \\
& miR204-R & 5'-ACCGTGTCGTGGAGTCGGCAATT-3' \\
& $\beta$ actin-R & 5'-ATGGTGGGTATGGGTCAGAAGG-3' \\
& $\beta$ actin-F & 5'-TGGCTGGGGTGTTGAAGGTC-3' \\
\hline
\end{tabular}




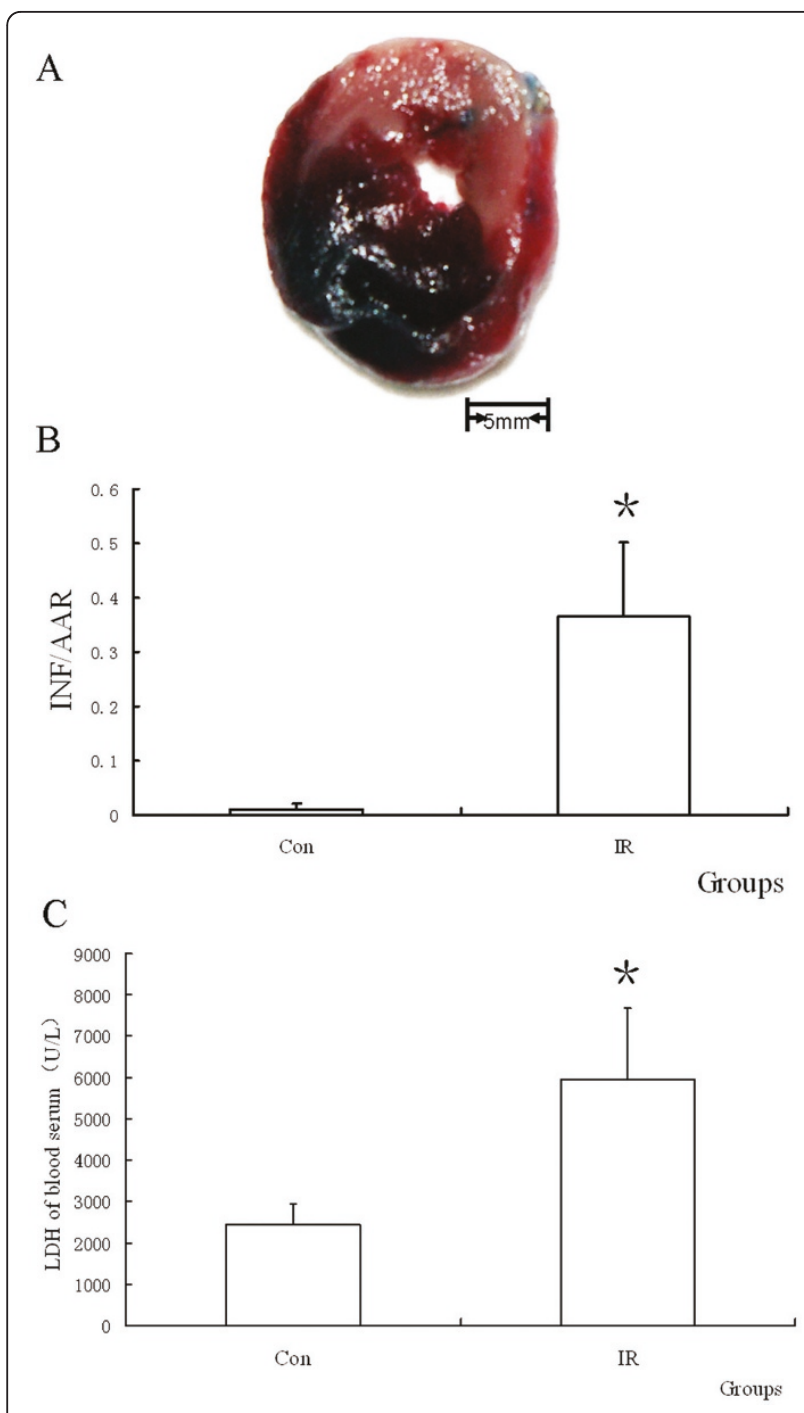

Figure 1 The heart injury induced by IR. (A) Representative midmyocardial crosssections of TTC-stained hearts for IR. Dark blue area, nonischemic zone; red area, area at risk, AAR; white area, infracted tissue. (B) The ratio of INF/AAR. It was found that IR increased the relative INF size compared with Con group. $\left(n=10,{ }^{*} \mathrm{P}=0.000<\right.$ 0.05, compared with Con group). (C) LDH assay of blood serum. The activitie of LDH was increased by IR compared with Con group. (n $=10,{ }^{*} P=0.000<0.05$, compared with Con group).

group and IR group $(\mathrm{n}=10)$, and found that it was enhanced by IR. (Figure 3)

\section{Discussion}

In recent years, autophagy has attracted great interest because it was involved in many physiological processes. If autophagy destroys the cytosol and organelles beyond a certain threshold, autophagic cell death will occur [25]. Autophagy was detrimental during reperfusion although it protected the cardiomyocytes during ischemia, which has been demonstrated by Matsui and his

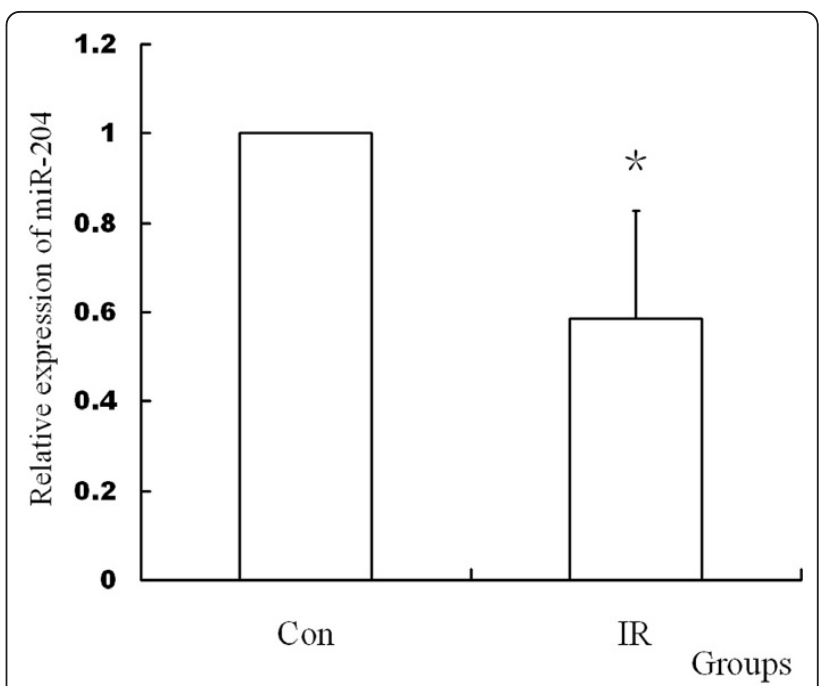

Figure 2 Results of miR-204 expression with RT-PCR after IR injury. It was found that miR-204 was down-regulated by IR. ( $n=$ $10, P=0.026<0.05)$

collaborators [26]. It has been reported that autophagy contributed to cell death when apoptosis is inhibited, and sometimes the early stages of autophagy were required for apoptosis [24]. LC3-II was the marker of autophagosome, instead of LC3-I. So the ratio of LC3II/LC3-I could stand for the level of autophagy. In our study, we found that IR up-regulated the protein expression of LC3-II together with increasing the ratio of

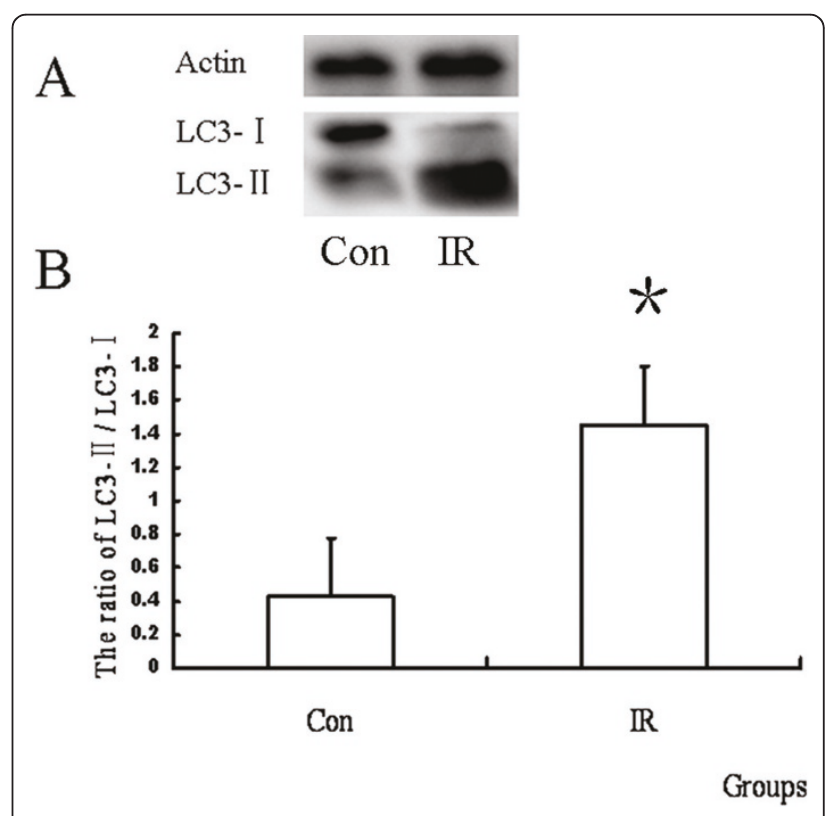

Figure 3 Results of LC3 protein expression with western blot after IR injury. (A) Representative western blot of LC3 from different groups. (B) The ratio of LC3-II/LC3-I in different groups. It was found that LC3-II was up-regulated by IR. $\left(n=10,{ }^{*} P=0.000<0.05\right)$. 
autophagy cell. So it would be beneficial for the revascularized hearts, to find a method of regulating LC3-II expression.

MiRNA is a group of small, non-coding RNAs which regulates gene expression in a sequence-dependent manner. They are endogenous regulators of gene expression, and have been demonstrated to be involved in cardiac IR injury. According to the bioinformatics of Targetscan, miR-204, which has anti-apoptosis effect [23], may regulate the expression of LC3-II.

In our study, it was found that IR could down-regulate miR-204 together with up-regulated LC3-II protein. When miR-204 mimic was transferred into cardiomyocytes, LC3-II protein was attenuated, and LC3-II protein was up-regulated by AMO-204 which was concentrationdependented as other miRNAs [27]. But LC3-I was not regulated by miR-204, as the previous study [28]. These results demonstrated that miR-204 may regulate cardiomyocytes autophagy through LC3-II during IR injury.

\section{Conclusions}

Our results demonstrated that miR-204 played an important role by regulating LC3-II protein during IR. So it became possible to control the autophagy under a beneficial threshold by regulating miR-204 expression, for protecting the cardiomyocyte against IR injury. But it is still unknown how did IR regulated the expression of miR-204, and this will be our next work.

\section{Acknowledgements}

This work was supported by the National Nature Science Foundation of China (No.30901470, No.30800375 and No.30700157).

\section{Author details \\ 'Department of Cardiothoracic Surgery, Changzheng Hospital, the second military medical university, Fengyang road 415\#, shanghai, China, 200003. ${ }^{2}$ Department of Physiology, the second military medical university, Xiangyin road 800\#, Shanghai, China, 200433. ${ }^{3}$ Department of Anesthesiology, Xinhua Hospital, Shanghai Jiaotong University School of Medicine, Kongjiang road 1665\#, Shanghai, China, 200092.}

\section{Authors' contributions}

Jian Xiao carried out the IR model, RT-PCR and Western-blot studies, and drafted the manuscript. Xiaoyan Zhu carried out the miR-204 transferring into cardiomyocytes, and participated in the sequence alignment of miR-204 mimic and AMO-204. Bin He carried out the primers synthesis of miR-204 and participated in drafting the manuscript. Bo Kang carried out the cardiomyocytes culture. Yufeng Zhang carried out the LDH assay. Zhinong Wang and Xin Ni conceived of the study, and participated in its design and coordination. All authors read and approved the final manuscript.

\section{Competing interests}

The authors declare that they have no competing interests.

Received: 16 January 2011 Accepted: 1 June 2011

Published: 1 June 2011

\section{References}

1. Melendez A, Talloczy Z, Seaman M, Eskelinen EL, Hall DH, Levine B: Autophagy genes are essential for dauer development and life-span extension in c. Elegans. Science 2003, 301:1387-1391.
2. Stromhaug PE, Klionsky DJ: Approaching the molecular mechanism of autophagy. Traffic 2001, 2:524-531.

3. Otto GP, Wu MY, Kazgan N, Anderson OR, Kessin RH: Macroautophagy is required for multicellular development of the social amoeba dictyostelium discoideum. J Biol Chem 2003, 278:17636-17645.

4. Yan L, Vatner DE, Kim SJ, Ge H, Masurekar M, Massover WH, Yang G, Matsui Y, Sadoshima J, Vatner SF: Autophagy in chronically ischemic myocardium. Proc Natl Acad Sci USA 2005, 102:13807-13812.

5. Zhang $Y$, Sun $Q$, He B, Xiao J, Wang Z, Sun X: Anti-inflammatory effect of hydrogen-rich saline in a rat model of regional myocardial ischemia and reperfusion. Int J Cardiol 2010.

6. Gustafsson AB, Gottlieb RA: Recycle or die: The role of autophagy in cardioprotection. J Mol Cell Cardiol 2008, 44:654-661.

7. Zhang JL, Lu JK, Chen D, Cai Q, Li TX, Wu LS, Wu XS: Myocardial autophagy variation during acute myocardial infarction in rats: The effects of carvedilol. Chin Med J (Engl) 2009, 122:2372-2379.

8. Cuervo AM: Autophagy: Many paths to the same end. Mol Cell Biochem 2004, 263:55-72.

9. Hein S, Arnon E, Kostin S, Schonburg M, Elsasser A, Polyakova V, Bauer EP, Klovekorn WP, Schaper J: Progression from compensated hypertrophy to failure in the pressure-overloaded human heart: Structural deterioration and compensatory mechanisms. Circulation 2003, 107:984-991.

10. Decker RS, Wildenthal K: Lysosomal alterations in hypoxic and reoxygenated hearts. I. Ultrastructural and cytochemical changes. Am J Pathol 1980, 98:425-444.

11. Sadoshima J: The role of autophagy during ischemia/reperfusion. Autophagy 2008, 4:402-403.

12. Nishida K, Kyoi S, Yamaguchi O, Sadoshima J, Otsu K: The role of autophagy in the heart. Cell Death Differ 2009, 16:31-38.

13. Kabeya Y, Mizushima N, Ueno T, Yamamoto A, Kirisako T, Noda T, Kominami E, Ohsumi Y, Yoshimori T: LC3, a mammalian homologue of yeast apg8p, is localized in autophagosome membranes after processing. EMBO J 2000, 19:5720-5728

14. Asanuma K, Tanida I, Shirato I, Ueno T, Takahara H, Nishitani T, Kominami E, Tomino Y: Map-Ic3, a promising autophagosomal marker, is processed during the differentiation and recovery of podocytes from pan nephrosis. Faseb J 2003, 17:1165-1167.

15. Huang ZP, Neppl RL, Wang DZ: Micrornas in cardiac remodeling and disease. J Cardiovasc Transl Res 2010, 3:212-218.

16. Ye $Y, H u Z$, Lin $Y$, Zhang C, Perez-Polo JR: Downregulation of microrna-29 by antisense inhibitors and a ppar-gamma agonist protects against myocardial ischaemia-reperfusion injury. Cardiovasc Res 2010, 87:535-544.

17. Roy S, Khanna S, Hussain SR, Biswas S, Azad A, Rink C, Gnyawali S, Shilo S, Nuovo GJ, Sen CK: Microrna expression in response to murine myocardial infarction: Mir-21 regulates fibroblast metalloprotease- 2 via phosphatase and tensin homologue. Cardiovasc Res 2009, 82:21-29.

18. Pan ZW, Lu YJ, Yang BF: Micrornas: A novel class of potential therapeutic targets for cardiovascular diseases. Acta Pharmacol Sin 2010, 31:1-9.

19. Bostjancic E, Zidar N, Stajer D, Glavac D: Micrornas mir-1, mir-133a, mir$133 \mathrm{~b}$ and mir-208 are dysregulated in human myocardial infarction. Cardiology 2010, 115:163-169.

20. Xu C, Lu Y, Pan Z, Chu W, Luo X, Lin H, Xiao J, Shan H, Wang Z, Yang B: The muscle-specific micrornas mir-1 and mir-133 produce opposing effects on apoptosis by targeting hsp60, hsp70 and caspase- 9 in cardiomyocytes. J Cell Sci 2007, 120:3045-3052.

21. Ren XP, Wu J, Wang X, Sartor MA, Qian J, Jones K, Nicolaou P, Pritchard TJ, Fan GC: Microrna-320 is involved in the regulation of cardiac ischemia/ reperfusion injury by targeting heat-shock protein 20. Circulation 2009, 119:2357-2366

22. Rane $\mathrm{S}, \mathrm{He}$ M, Sayed D, Vashistha H, Malhotra A, Sadoshima J, Vatner DE, Vatner SF, Abdellatif M: Downregulation of mir-199a derepresses hypoxiainducible factor-1alpha and sirtuin 1 and recapitulates hypoxia preconditioning in cardiac myocytes. Circ Res 2009, 104:879-886.

23. Yang BF, Lu YJ, Wang ZG: Micrornas and apoptosis: Implications in the molecular therapy of human disease. Clin Exp Pharmacol Physiol 2009, 36:951-960.

24. Nishida K, Yamaguchi O, Otsu K: Crosstalk between autophagy and apoptosis in heart disease. Circ Res 2008, 103:343-351.

25. Rothermel BA, Hill JA: Autophagy in load-induced heart disease. Circ Res 2008, 103:1363-1369 
26. Matsui Y, Takagi H, Qu X, Abdellatif M, Sakoda H, Asano T, Levine B, Sadoshima J: Distinct roles of autophagy in the heart during ischemia and reperfusion: Roles of amp-activated protein kinase and beclin 1 in mediating autophagy. Circ Res 2007, 100:914-922.

27. He B, Xiao J, Ren AJ, Zhang YF, Zhang H, Chen M, Xie B, Gao XG, Wang YW: Role of mir-1 and mir-133a in myocardial ischemic postconditioning. J Biomed Sci 2011, 18:22.

28. Jian X, Xiao-yan Z, Bin H, Yu-feng Z, Bo K, Zhi-nong W, Xin N: MiR-204 regulate cardiomyocyte autophagy induced by hypoxia-reoxygenation through LC3-II. Int J Cardiol 2011, 148(1):110-2.

doi:10.1186/1423-0127-18-35

Cite this article as: Xiao et al:: MiR-204 regulates cardiomyocyte autophagy induced by ischemia-reperfusion through LC3-II. Journal of Biomedical Science 2011 18:35.

Submit your next manuscript to BioMed Central and take full advantage of:

- Convenient online submission

- Thorough peer review

- No space constraints or color figure charges

- Immediate publication on acceptance

- Inclusion in PubMed, CAS, Scopus and Google Scholar

- Research which is freely available for redistribution

Submit your manuscript at www.biomedcentral.com/submit 